\title{
RECOMBINING PLASMA IN THE GAMMA-RAY-EMITTING MIXED-MORPHOLOGY SUPERNOVA REMNANT 3C 391
}

\author{
T. Ergin ${ }^{1,2}$, A. Sezer ${ }^{1,2}$, L. Saha ${ }^{3}$, P. Majumdar ${ }^{3}$, A. Chatterjez ${ }^{3}$, A. Bayirli ${ }^{2}$, and E. N. Ercan ${ }^{2}$ \\ ${ }^{1}$ TUBITAK Space Technologies Research Institute, ODTU Campus, 06531 Ankara, Turkey; tulun.ergin@tubitak.gov.tr \\ ${ }^{2}$ Physics Department, Bogazici University, Bebek, 34342 Istanbul, Turkey \\ ${ }^{3}$ Saha Institute of Nuclear Physics, Kolkata, West Bengal 700064, India \\ Received 2013 November 4; accepted 2014 June 6; published 2014 July 3
}

\begin{abstract}
A group of middle-aged mixed-morphology (MM) supernova remnants (SNRs) interacting with molecular clouds (MCs) has been discovered to be strong GeV gamma-ray emitters by the Large Area Telescope (LAT) on board the Fermi Gamma-Ray Space Telescope (Fermi-LAT). The recent observations of the Suzaku X-ray satellite have revealed that some of these interacting gamma-ray-emitting SNRs, such as IC443, W49B, W44, and G359.1-0.5, have overionized plasmas. 3C 391 (G31.9+0.0) is another Galactic MM SNR interacting with MCs. It was observed in $\mathrm{GeV}$ gamma rays by Fermi-LAT as well as in the $0.3-10.0 \mathrm{keV}$ X-ray band by Suzaku. In this work, 3C 391 was detected in $\mathrm{GeV}$ gamma rays with a significance of $\sim 18 \sigma$ and we showed that the $\mathrm{GeV}$ emission is point-like in nature. The GeV gamma-ray spectrum was shown to be best explained by the decay of neutral pions assuming that the protons follow a broken power-law distribution. We revealed radiative recombination structures of silicon and sulfur from 3C 391 using Suzaku data. In this paper, we discuss the possible origin of this type of radiative plasma and hadronic gamma rays.
\end{abstract}

Key words: gamma rays: ISM - ISM: clouds - ISM: individual objects (G31.9+0.0, 3C391) - ISM: supernova remnants - X-rays: ISM

Online-only material: color figures

\section{INTRODUCTION}

About $10 \%$ of all Galactic supernova remnants (SNRs) have radio shells and center-filled thermal X-rays; these SNRs are called mixed-morphology (MM) SNRs (Rho \& Petre 1998). The first SNRs detected by the Fermi Gamma-Ray Space Telescope (Fermi-LAT) were mostly middle-aged MM SNRs interacting with molecular clouds (MCs) and exhibiting $\mathrm{GeV}$ gamma-ray luminosities distinctively higher than those of other detected SNRs, i.e., $\sim 10^{35}-10^{36} \mathrm{erg} \mathrm{s}^{-1}$ for IC443, W28, W51C, W44, and W49B (Abdo et al. 2010b, 2010c, 2009b, 2010d, 2010e; Castro \& Slane 2010). Interactions of these MM SNRs with MCs was shown by $1720 \mathrm{MHz} \mathrm{OH}$ masers (Yusef-Zadeh et al. 1995; Frail et al. 1996; Green et al. 1997; Claussen et al. 1997; Hewitt \& Yusef-Zadeh 2009) and near-infrared observations (Keohane et al. 2007).

MM SNRs interacting with MCs are interesting targets for the detection of gamma rays of hadronic origin, which provides clear evidence that these SNRs are sites of proton acceleration (Ackermann et al. 2013). The hadronic mechanism is the production of two gamma rays from the decay of a neutral pion created in a proton-proton interaction during the passage of SNR shocks through the dense molecular material around the source. Therefore, the gamma-ray spectra of these SNRs rise steeply below $250 \mathrm{MeV}$ and, at energies greater than $1 \mathrm{GeV}$, they trace the parent proton energy distribution (Ackermann et al. 2013). Interactions with MCs may hint that MM SNRs are associated with star-forming regions containing massive stars with strong stellar winds surrounded by circumstellar matter (CSM) and these massive stars are possibly the progenitors of MM-type remnants. When the supernova (SN) blast wave breaks out of the CSM into the interstellar medium (ISM), its velocity rapidly rises and the particle acceleration increases. Shimizu et al. (2012) calculated the amount of gamma-ray emission from an SNR blast wave breaking out of the CSM.

In young SNRs, shocks create an X-ray-emitting plasma, called ionizing plasma (IP), where the thermal energy of electrons $\left(k T_{e}\right)$ is higher than the ionization energy $\left(k T_{z}\right)$. This plasma gradually reaches collisional ionization equilibrium (CIE), where an equilibrium state between recombination and ionization is established $\left(k T_{e}=k T_{z}\right)$. X-ray studies of $A S C A$ on six MM SNRs (Kawasaki et al. 2002, 2005) revealed the existence of recombining plasma (RP) for IC443 and W49B, where $k T_{z}$ is higher than the $k T_{e}$. Recently, the X-ray Imaging Spectrometer (XIS) (Koyama et al. 2007) on board Suzaku (Mitsuda et al. 2007) has discovered strong radiative recombination continuum (RRC) features from six MM SNRs, i.e.,IC443, W49B, G359.1-0.5, W28, W44, and G346.6-0.2 (Yamaguchi et al. 2009; Ozawa et al. 2009; Ohnishi et al. 2011; Sawada \& Koyama 2012; Uchida et al. 2012; Yamauchi et al. 2013). There are two main scenarios describing the origin of the (electron cooling in) recombining (overionized) plasma in SNRs.

1. Thermal conduction. When the hot ejecta inside the SNR interior, which is in the form of normal IP or CIE plasma, encounters cold MCs, the electron energy will be transferred to the MCs by thermal conduction and the electron temperature falls rapidly (Cox et al. 1999; Shelton et al. 1999; Orlando et al. 2008). This condition then forms the RP.

2. Adiabatic cooling. If the CSM surrounding a progenitor is dense enough, CIE plasma will be formed at the early stages of the evolution of an SNR. When the blast wave breaks out of the dense CSM and expands rapidly into the rarefied ISM, the electron temperature drops due to fast cooling by adiabatic expansion, which results in RP (Itoh \& Masai 1989; Moriya 2012; Shimizu et al. 2012). 
Recently, Lopez et al. (2013) produced overionization maps across all SNRs to distinguish between the two RP scenarios in W49B.

The Galactic SNR 3C 391 (G31.9+0.0), a member of the MM class, was suggested to be a result of an asymmetric core-collapse SN explosion of a massive $\left(\gtrsim 25 M_{\odot}\right)$ progenitor star (Su \& Chen 2005). H I absorption measurements by Radhakrishnan et al. (1972) show that the distance to 3C 391 is at least $7.2 \mathrm{kpc}$ (assuming a Galactocentric radius of $8.5 \mathrm{kpc}$ ) and for emission without absorption, this indicates an upper limit of $11.4 \mathrm{kpc}$.

In the radio band, 3C 391 was observed by the Very Large Array (Reynolds \& Moffett 1993; Moffett \& Reynolds 1994) as a partial shell of $5^{\prime}$ radius with a breakout morphology, where the intensity of the radio emission in the shell rises in the bright northwest rim (NW) and drops and vanishes toward the southeast rim (SE). The $\mathrm{CO}(1-0)$ line observations of $3 \mathrm{C}$ 391 by Wilner et al. (1998) showed that 3C 391 is embedded in the edge of an MC, supporting the idea that the progenitor has exploded within the MC and that the $\mathrm{SN}$ blast wave has now broken out through the cloud boundary. Indirect evidence for 3C 391 expanding into a medium with a different gas density comes from X-rays. In the X-ray band, 3C 391 was observed with Einstein (Wang \& Seward 1984), ROSAT (Rho \& Petre 1996), Chandra (Chen et al. 2004), and ASCA (Chen \& Slane 2001; Kawasaki et al. 2005). ROSAT and Einstein data revealed two bright X-ray peaks within the SNR: a brighter X-ray peak toward the interior of the weak SE radio rim and a fainter one in the interior of the bright NW radio shell.

Using ROSAT observations, Rho \& Petre (1996) applied a single-temperature thermal model and obtained an absorbing column density of $N_{\mathrm{H}} \sim 2.4 \times 10^{22} \mathrm{~cm}^{-2}$ and an electron temperature of $k T_{e} \sim 0.5 \mathrm{keV}$. They also found enhanced abundances of Mg, Si, and S. Chen et al. (2004) found that the Xray spectra obtained from Chandra data can be best described by the non-equilibrium ionization collisional plasma (VNEI) model. The spectral fits showed that the diffuse emission has ionization parameters $\left(\tau=n_{e} t\right)$ close to or higher than $10^{12}$ $\mathrm{cm}^{-3} \mathrm{~s}$. They concluded that the hot plasma in the SNR is very close to or in the ionization equilibrium. They found an electron temperature of $\sim 0.5-0.6 \mathrm{keV}$ and estimated an age of $\sim 4 \times 10^{3}$ yr for the remnant. From ASCA observational data, Kawasaki et al. (2005) found an electron temperature value of $\sim 0.53 \mathrm{keV}$ by applying a non-equilibrium ionization (NEI) model to the spectra. They obtained an ionization timescale of $\tau \sim 2.5 \times$ $10^{12} \mathrm{~cm}^{-3} \mathrm{~s}$, suggesting that the plasma has reached ionization equilibrium.

Frail et al. (1996) observed two $\mathrm{OH}$ masers at $1720 \mathrm{MHz}$ with velocities of 105 and $110 \mathrm{~km} \mathrm{~s}^{-1}$, coincident with the southeast and northeast rims of 3C 391, respectively, showing the first clear evidence for 3C 391 interacting with an MC. The $\mathrm{CO}(1-0)$ data support the evidence of SNR-MC interaction (Wilner et al. 1998). Further evidence for shock interactions are obtained through CS line observations by Reach \& Rho (1999), measurements of strongly enhanced [O I] $63 \mu \mathrm{m}$ (Reach \& Rho 1996) at the NW rim of 3C 391, and recent $\mathrm{OH}$ maser observations by Hewitt et al. (2008).

3C 391 was observed in $\mathrm{GeV}$ gamma rays by Fermi-LAT (Atwood et al. 2009) and it was listed in the Fermi-LAT twoyear point-source catalog (2FGL; Nolan et al. 2012) as a pointsource, called 2FGL J1849.3-0055. Castro \& Slane (2010) analyzed the $\mathrm{GeV}$ data of $3 \mathrm{C} 391$ and reported a $\sim 12 \sigma$ detection. They showed that the peak of the significance map was shifted $4^{\prime}$ away from the NW edge of the radio shell. The spectrum of 3C 391 was best described as a power-law (PL) model with a spectral index of $\Gamma=-2.33 \pm 0.11$. They found an integrated flux of $3 \mathrm{C} 391$ as $F(0.1-100 \mathrm{GeV})=(1.58 \pm 0.26) \times$ $10^{-7}$ photons $\mathrm{cm}^{-2} \mathrm{~s}^{-1}$, Castro \& Slane (2010). At TeV energies, H.E.S.S. reported integral flux upper limits at the 95\% CL in units of the flux of the Crab nebula as $0.8 \mathrm{Crab}$ units, Bochow (2011).

The gamma-ray emission from 3C 391 might be the result of hadronic interactions between the SNR shock and the associated MC. To understand if this is the case, we have performed a detailed modeling of the $\mathrm{GeV}$ gamma-ray spectrum. We have investigated the gamma-ray source morphology and variability. Moreover, we investigated the characteristics of the continuum radiation, thermal bremsstrahlung continuum, or RRC by utilizing the superior spectral capabilities for diffuse sources of XIS on board Suzaku. We report on our results of RP in 3C 391 and discuss different scenarios for its origin.

\section{DATA ANALYSIS AND RESULTS}

\subsection{Gamma Rays}

We analyzed the 3C 391 Fermi-LAT data taken in the period between 2008 August 4 and 2013 August 18. The events data were taken from a circular region of interest (ROI) with a radius of $18^{\circ}$ centered at the position of R.A. $(\mathrm{J} 2000)=18^{\mathrm{h}} 49^{\mathrm{m}} 26^{\mathrm{s}} \cdot 40$ and decl. (J2000) $=-00^{\circ} 55^{\prime} 37^{\prime \prime} .20$ and the events suggested for Fermi-LAT Pass 7 for galactic point source analysis type were selected using gtselect of the Fermi Science Tools (FST). To prevent event contamination at the edge of the field of view caused by the bright gamma rays from the Earth's limb, we cut out the gamma rays with reconstructed zenith angles greater than $105^{\circ}$. For the rest of the analysis, we implemented pointlike (Kerr 2011; Lande et al. 2012) (FST-v9r32p0) and the standard binned likelihood analysis tools (FST-v9r27p1), both based on the gtlike, to cross-check the validity of the results. The analysis was performed within a square region of $\sim 25^{\circ} \times 25^{\circ}$. The gamma-ray events in the data were binned in energy at 15 logarithmic steps between $250 \mathrm{MeV}$ and $300 \mathrm{GeV}$. For the binned likelihood analysis (Abdo et al. 2009a), the matching energy-dependent exposure maps were produced based on pointing direction, orientation, orbit location, and livetime accumulation of Large Area Telescope (LAT). The pointspread function (PSF) of Fermi-LAT is up to $3^{\circ}$ at $100 \mathrm{MeV}$ and 0.1 above $10 \mathrm{GeV}$. The large PSF of LAT means that, at low energies, sources from outside the ROI can affect the analyzed source. To compensate for this and to ensure that the exposure map accounts for contributions from all the sources in the analysis region, exposure maps were created such that they included sources up to $10^{\circ}$ outside the ROI. In addition, since at low energies the PSF is large, the exposure map should be expanded by another $10^{\circ}$ to accommodate this additional exposure, Abdo et al. (2009a).

The spectral properties of the gamma-ray emission were studied by comparing the observation with models of possible sources in the ROI. Predictions were made by convolving the spatial distribution and spectrum of the source models with the instrument response function (IRF) and with the exposure of the observation. In the analysis, we used the IRF version P7SOURCE_V6.

The model of the analysis region contains the diffuse background sources and all the point-like sources from the 2FGL located within a distance of $18^{\circ}$ from the ROI center. We fixed 


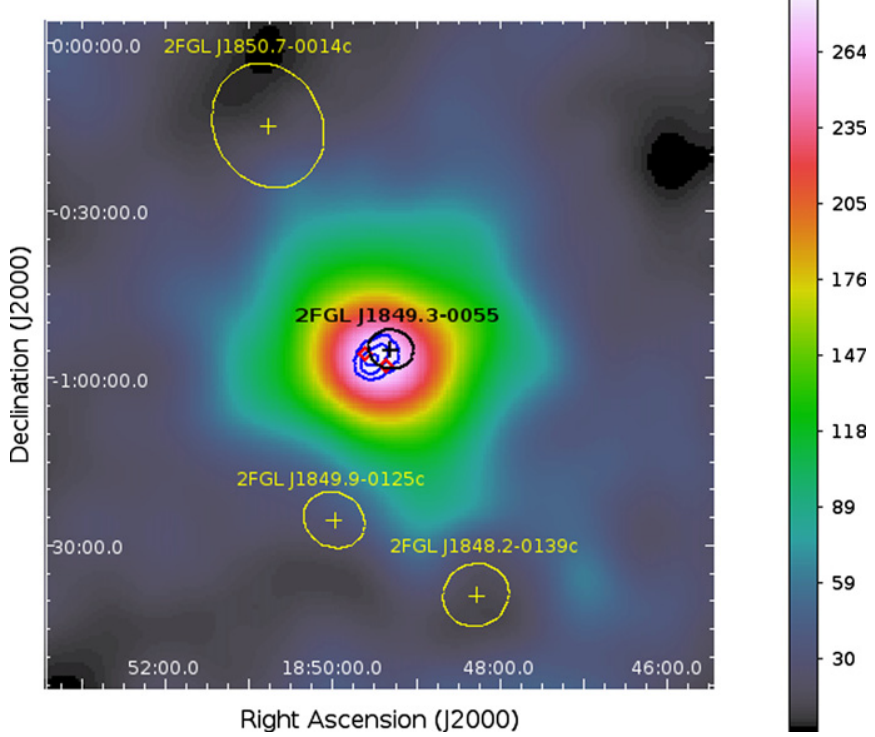

Figure 1. Gamma-ray TS map of the 3C 391 neighborhood with a bin size of $0.01 \times 0.01$. The blue contours show the Suzaku data from Figure 6, where three contours represent 14, 29, 43 counts. The yellow crosses and circles represent the 2FGL catalog sources and the black cross and circle is the GeV source from the 2FGL catalog corresponding to SNR 3C 391. The two red diamonds represent the two masers detected by Frail et al. (1996).

(A color version of this figure is available in the online journal.)

all parameters of the point-like sources in the model, except for three sources (shown in Figure 1 with yellow markers) within the distance of $2^{\circ}$ from the best-fit location of 3C 391, where we set their normalization and spectral parameters free. The standard diffuse background model has two components: the diffuse Galactic emission ( $g a l_{-} 2 y e a r p 7 v 6_{-} v 0$.fits) and the isotropic component (iso_p7v6source.txt), which is a sum of the extragalactic background, unresolved sources, and instrumental background. The normalization of the galactic diffuse background is set free during the analysis. The normalization of the isotropic component is fixed to one due to the difficulty disentangling it from Galactic interstellar emission over limited regions.

The background and source modeling were done by the binned likelihood analysis using gtlike of FST. To determine the best set of spectral parameters of the fit, we vary the parameters until the maximum likelihood is maximized. The detection of the source in this analysis is given by the test statistic (TS) value, where larger TS values indicate that the null hypothesis (maximum likelihood value for a model without an additional source) is incorrect. This means that the detection significance is approximately equal to the square root of the TS.

\subsubsection{Location and Spectrum}

Using both FST binned likelihood (with FST-v9r27p1) and pointlike (with FST-v9r32p0) analysis, we detected 3C 391 with a significance of $\sim 18 \sigma$. We computed the best-fit position within the ROI of 3C 391, which was found to be longitude $l=$ $31.879 \pm 0.022$ and latitude $b=0.022 \pm 0.022$. This best-fit position $^{4}$ enhanced the TS by $2.58 \sigma$ over the position of $2 \mathrm{FGL}$ J1849.3-0055 in the 2FGL. Then the model was refitted using the best-fit position to compute the TS map (Section 2.1.2) and the spectrum.

\footnotetext{
4 R.A. $(\mathrm{J} 2000)=18^{\mathrm{h}} 49^{\mathrm{m}} 26^{\mathrm{s}} 34$ and decl. $(\mathrm{J} 2000)=-00^{\circ} 55^{\prime} 37^{\prime \prime} .35$.
}

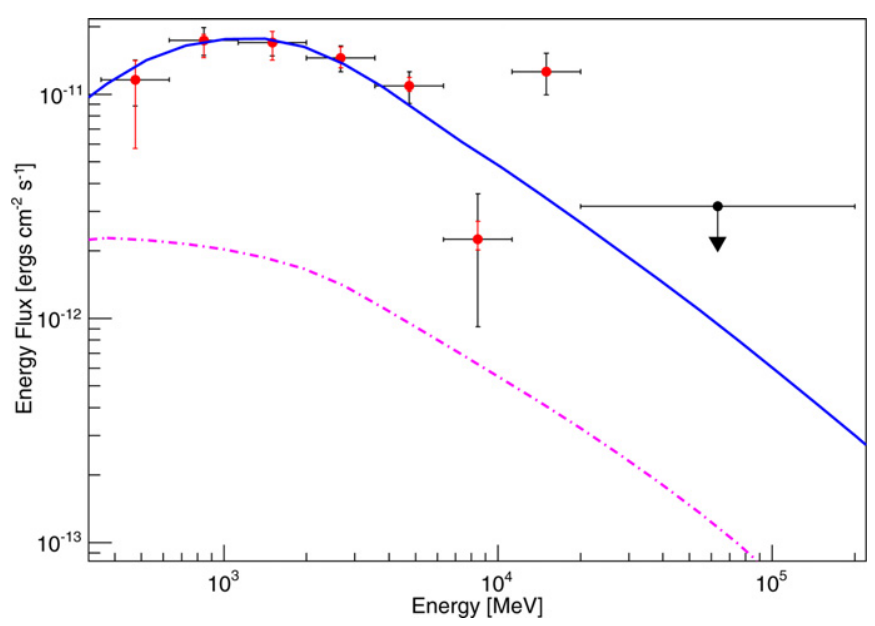

Figure 2. Gamma-ray SED of 3C 391, where the Fermi-LAT spectral data points are represented with red filled circles and their corresponding statistical and systematic errors are shown as black and red lines, respectively. The thick blue line passing through the data points shows the hadronic model fit to the data. The dashed-dotted magenta line represents the bremsstrahlung spectrum. The parameters used to estimate the emission spectra for both hadronic and leptonic models are mentioned in Section 2.1.5.

(A color version of this figure is available in the online journal.)

Table 1

Spectral Fit Parameters for PL, LP, and BPL between $250 \mathrm{MeV}$ and $300 \mathrm{GeV}$ Assuming 3C 391 as a Point-like Source

\begin{tabular}{lccccc}
\hline \hline $\begin{array}{l}\text { Spectral } \\
\text { Model }\end{array}$ & $\begin{array}{c}\text { Photon Flux } \\
\left(10^{-8} \text { photon } \mathrm{cm}^{-2} \mathrm{~s}^{-1}\right)\end{array}$ & $\Gamma_{1}$ & $\Gamma_{2}$ & $\begin{array}{c}E_{b} \\
(\mathrm{MeV})\end{array}$ & TS \\
\hline PL & $15.0 \pm 1.7$ & $2.30 \pm 0.03$ & $\ldots$ & $\ldots$ & 337 \\
LP & $7.14 \pm 0.34$ & $2.27 \pm 0.04$ & $0.15 \pm 0.45$ & 2430 & 337 \\
BPL & $4.89 \pm 0.57$ & $1.28 \pm 0.50$ & $2.50 \pm 0.04$ & 1060 & 338 \\
\hline
\end{tabular}

The observed spectral energy distribution (SED) of 3C 391 is shown in Figure 2, where the data points are represented by red filled circles and their corresponding statistical and systematic errors are shown with black and red lines, respectively. To check the functional form of the spectrum, we first considered 3C 391 as a point-like source. First, the PL function was fitted to the data between $250 \mathrm{MeV}$ and $300 \mathrm{GeV}$, but we noticed that the spectrum deviates from a PL function. So, we checked if the gamma-ray emission is better described by a log-parabola (LP) or a broken power-law (BPL) function, where the functional forms are as follows:

1. Log parabola:

$$
F(E)^{\mathrm{LP}}=N_{\circ}\left(E / E_{b}\right)^{\left(\Gamma_{1}+\Gamma_{2} \ln \left(E / E_{b}\right)\right)} \text {. }
$$

2. Broken power law:

$$
\begin{aligned}
F(E)^{\mathrm{BPL}} & =N_{\circ}\left(E / E_{b}\right)^{-\Gamma_{1}} \text { for } E<E_{b} \\
& =N_{\circ}\left(E / E_{b}\right)^{-\Gamma_{2}} \text { for } E>E_{b} .
\end{aligned}
$$

The results from these spectral fits are summarized in Table 1. Using different functions in fitting the spectrum of 3C 391, the likelihood ratio, TS, was used as a measure of the improvement of the likelihood fit with respect to the simple PL. The TS values of 337, 337, and 338 were found for PL, LP, and BPL fits, respectively.

The PL resulted in a spectral index of $\Gamma_{1}=2.30 \pm 0.03$, which is in agreement with the best-fit PL index value given for 3C 391 in the 2FGL ( 2.19), Nolan et al. (2012). This result also matches the results obtained by Castro \& Slane (2010), $\Gamma_{1}=2.33 \pm 0.11$. Additionally, the LP fit results (shown in Table 1) were found to be in good agreement with the results 
in 2FGL (Nolan et al. 2012), which are $\Gamma_{1}=2.35 \pm 0.16$ and $\Gamma_{2}=0.308 \pm 0.099$ for a fixed $E_{b}$ value of $2430 \mathrm{MeV}$.

The best-fit parameters for the BPL fit are $N_{\circ}=(1.15 \pm$ $0.69) \times 10^{-11} \mathrm{MeV}^{-1} \mathrm{~cm}^{-2} \mathrm{~s}^{-1}, \Gamma_{1}=1.28 \pm 0.50$, and $\Gamma_{2}$ $=2.50 \pm 0.04$, where the given uncertainties are statistical. The total energy flux was found to be $(6.28 \pm 0.16) \times$ $10^{-11} \mathrm{erg} \mathrm{cm}^{-2} \mathrm{~s}^{-1}$ with $E_{b}=1060 \pm 250 \mathrm{MeV}$.

Apart from the statistical uncertainties, there are systematic errors originating from the uncertainty of the Galactic diffuse background intensity. In order to calculate these systematic errors, we followed the prescriptions of Abdo et al. (2009b) and Castro et al. (2013) by varying the normalization value of the Galactic background by $\pm 6 \%$ from the best-fit value and use these new frozen values of the normalization parameter to recalculate the SED of 3C 391. The systematic errors on the SED are shown in Figure 2 in red on top of the statistical errors.

\subsubsection{Extension}

To investigate the morphology of 3 C 391 , we created a $2^{\circ} \times$ $2^{\circ}$ TS map of 3C 391 and its neighborhood with a bin size of $0.01 \times 0.01$. The TS map shown in Figure 1 was produced with pointlike using a background model file, which contained all the point-like sources and diffuse sources, but excluded 3C 391 from the model. So, it shows the TS distribution of gamma rays originating predominantly from 3C 391. In Figure 1, the blue contours represent the Suzaku XIS image in the $0.3-10.0 \mathrm{keV}$ energy band (from Figure 6), the yellow crosses and circles represent the 2FGL catalog sources and their statistical errors, respectively, and the black cross and circle represent the location and its statistical error of the GeV source from the 2FGL catalog corresponding to 3C 391, respectively. The peak value of the gamma-ray significance coincides with the X-ray remnant. The red diamonds indicate the locations of the two $\mathrm{OH}$ masers reported by Frail et al. (1996).

To search for the energy-dependent morphology, we split the data set into two energy ranges $(250 \mathrm{MeV}-1 \mathrm{GeV}$ and 1-300 GeV) and computed the TS maps for each energy range. We found no significant gamma-ray excess at the location of $3 \mathrm{C}$ 391 for the energy range between $250 \mathrm{MeV}$ and $1 \mathrm{GeV}$, but $3 \mathrm{C}$ 391 was detected in the higher-energy range of $1-300 \mathrm{GeV}$ with a significance of $\sim 15 \sigma$ using a BPL spectral model.

Additionally, using pointlike we have checked the extension of 3C 391 by fitting a disk template and assuming 3C 391 has a PL/BPL-type spectrum. To detect the extension of a source, we use the TS of the extension $\left(\mathrm{TS}_{\mathrm{ext}}\right)$ parameter, which is the likelihood ratio comparing the likelihood for being a point-like source $\left(L_{\mathrm{pt}}\right)$ to a likelihood for an existing extension $\left(L_{\mathrm{ext}}\right)$, $\mathrm{TS}_{\mathrm{ext}}=2 \log \left(L_{\mathrm{ext}} / L_{\mathrm{pt}}\right)$. pointlike calculates $\mathrm{TS}_{\mathrm{ext}}$ by fitting a source first with a disk template and then as a point-like source.

According to the extension studies by Lande et al. (2012), the extended source detection threshold is $\mathrm{TS}_{\text {ext }}=16$, where the threshold is defined as the source flux at which the value of $\mathrm{TS}_{\text {ext }}$ averaged over many statistical realizations is 16 . From simulation studies, it is found that to resolve a disklike extension of $r=0.1$ at the level of $\mathrm{TS}_{\mathrm{ext}}=16$, the source must have a minimum flux of $3 \times 10^{-7}$ photon $\mathrm{cm}^{-2} \mathrm{~s}^{-1}$ for a spectral index value of 2.0 and a flux of $2 \times 10^{-6}$ photon $\mathrm{cm}^{-2} \mathrm{~s}^{-1}$ for a spectral index value of 2.5 (Lande et al. 2012). So, for 3C 391 $\mathrm{TS}_{\mathrm{ext}}$ was found as 0.008 and 0.52 after a disk template fitting for 3C 391 having a PL- and BPL-type spectrum, respectively (Table 2). Both of the $\mathrm{TS}_{\text {ext }}$ values are smaller than 16, which indicates that a disklike extension with $r \sim 0$. 1 could not be resolved at the integrated flux level and spectral index values of

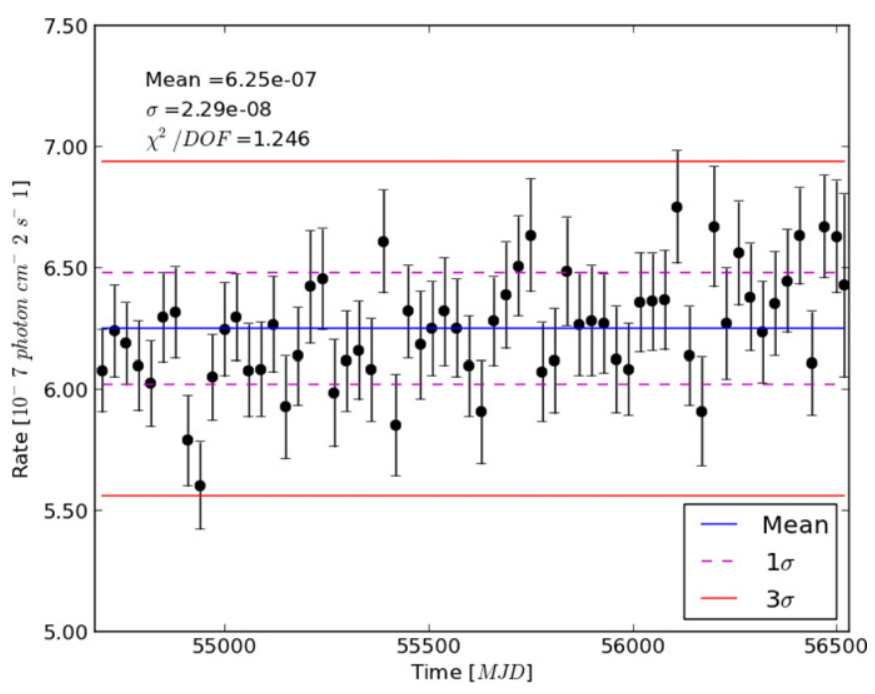

Figure 3. Monthly gamma-ray variability for 3C 391 with a BPL-fit spectrum in the energy range of $0.25-300 \mathrm{GeV}$.

(A color version of this figure is available in the online journal.)

Table 2

Fit Results of a Disklike Extension Model Applied to 3C 391 Gamma-ray Data between $250 \mathrm{MeV}$ and $300 \mathrm{GeV}$ for the PL- and BPL-type Spectral Models

\begin{tabular}{|c|c|c|c|c|}
\hline $\begin{array}{l}\text { Disk } \\
\text { Model }\end{array}$ & $\begin{array}{c}\text { Longitude } \\
\left(^{\circ}\right)\end{array}$ & $\begin{array}{l}\text { Latitude } \\
\left(^{\circ}\right)\end{array}$ & $\begin{array}{c}\text { Sigma } \\
\left({ }^{\circ}\right)\end{array}$ & $\mathrm{TS}_{\text {ext }}$ \\
\hline $\mathrm{PL}$ & $31.87 \pm 0.02$ & $0.023 \pm 0.017$ & $0.10 \pm 0.15$ & 0.008 \\
\hline BPL & $31.88 \pm 0.02$ & $0.028 \pm 0.017$ & $0.10 \pm 0.26$ & 0.52 \\
\hline
\end{tabular}

$1.5 \times 10^{-7}$ photon $\mathrm{cm}^{-2} \mathrm{~s}^{-1}$ and 2.3 for the PL-type spectrum and $4.9 \times 10^{-8}$ photon $\mathrm{cm}^{-2} \mathrm{~s}^{-1}$ and $2.5\left(\Gamma_{2}\right)$ for the BPL-type spectrum of $3 \mathrm{C} 391$.

\subsubsection{Variability and Pulsation}

Variability or pulsations can effect the analysis results for $3 \mathrm{C}$ 391. So, we checked for both of these effects in the data. We searched for long-term variability in the light curve of 3C 391 produced using the data from the circular region of $1^{\circ}$ around the best-fit position. Figure 3 shows the one-month binned light curve after fitting the spectrum with a BPL, where each flux point remains within $1 \sigma$ and $3 \sigma$. Fitting these flux points to a straight line (shown as a blue line in Figure 3), yields a $\chi^{2} /$ degrees of freedom (dof) of $\sim 1.25$. Thus, we conclude that there is no longterm variability observed in the close neighborhood of 3C 391.

We have also checked if the spectral shape of 3C 391 fits the standard spectrum of a pulsar, a Power Law with Exponential Cutoff (PLEC). The best-fit cutoff energy is found to be $28.80 \pm$ $6.73 \mathrm{GeV}$, which is an order of magnitude away from the range of typical pulsar cutoff energies (Abdo et al. 2010a). The PLEC fit did not show a significant improvement over the PL, BPL, and LP spectral fits.

\subsubsection{Molecular Environment}

To estimate the average density of the vicinity of 3C 391, we used the $\mathrm{CO}$ data of Harvard-Smithsonian Center for Astrophysics $1.2 \mathrm{~m}$ Millimeter-Wave Telescope (Dame et al. 1987). We analyzed the CO gas in the whole velocity range integrated from -50 to $120 \mathrm{~km} \mathrm{~s}^{-1}$, where the velocity intervals were divided such that each range included at least one cloud cluster peaking in temperature at a certain velocity. Figure 4 

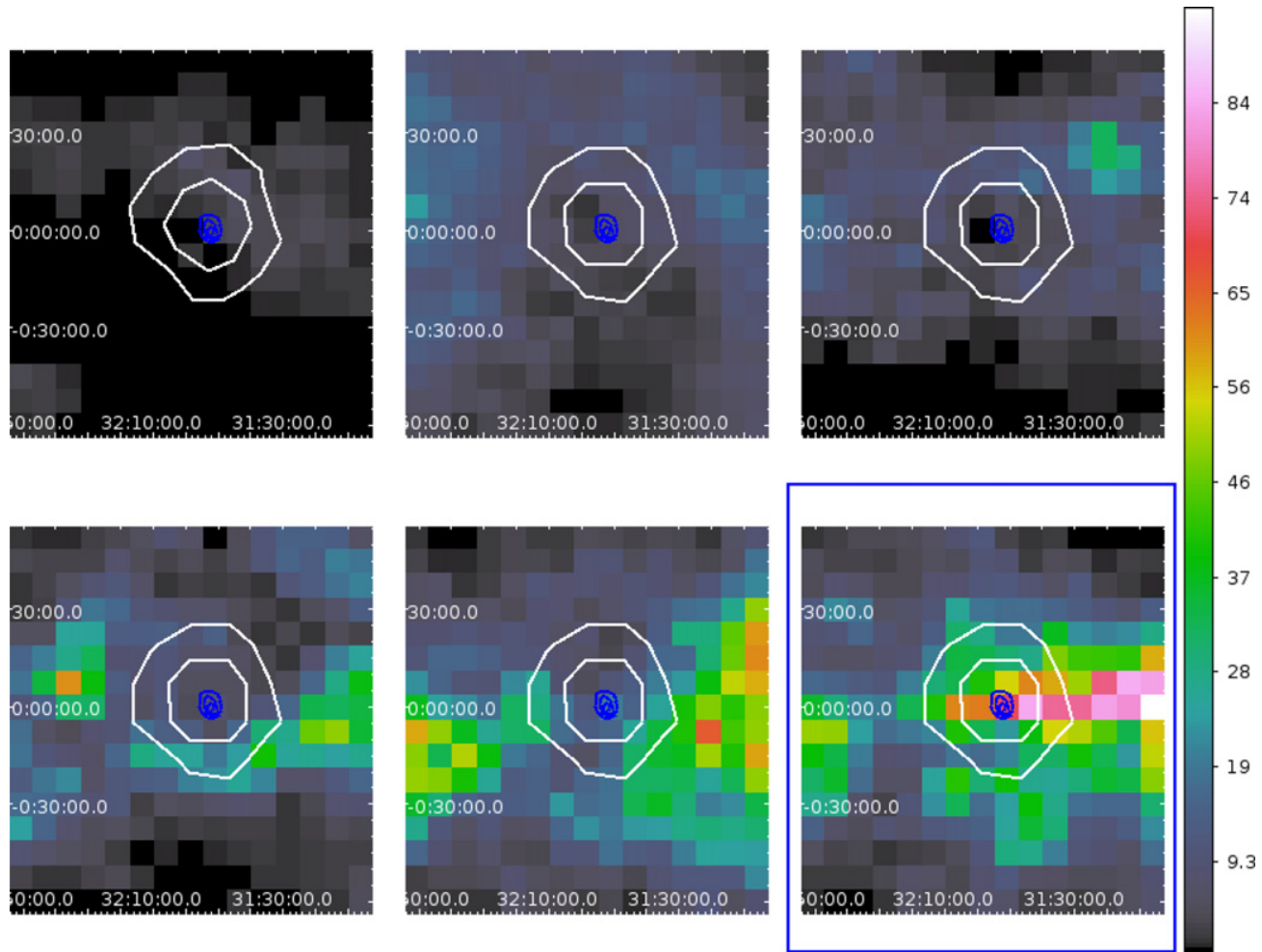

Figure 4. Maps in galactic coordinates (longitude $x$-axis and latitude $y$-axis) of the integrated CO intensity ( $W_{\mathrm{CO}}$ ) for six different velocity ranges (top, from left: $[-50,0],[0,15],[15,35] \mathrm{km} \mathrm{s}^{-1}$; bottom, from left: $[35,60],[60,90],[90,120] \mathrm{km} \mathrm{s}^{-1}$ ) at the location of $3 \mathrm{C} 391$ and its vicinity. For all maps, the range for $W_{\mathrm{CO}}$ is fixed between 0 and $92.8 \mathrm{~K} \mathrm{~km} \mathrm{~s}^{-1}$.

(A color version of this figure is available in the online journal.)

shows the $\mathrm{CO}$ maps produced at different velocity ranges of $[-50,0],[0,15],[15,35] \mathrm{km} \mathrm{s}^{-1}$ starting from the top left and $[35,60],[60,90],[90,120] \mathrm{km} \mathrm{s}^{-1}$ starting from the bottom left. The white contours represent the TS values of 3C 391 gammaray data at 41 and 83 , and the blue contours are the X-ray counts at 14,29 , and 43 .

The velocity integrated $\mathrm{CO}$ intensity $\left(W_{\mathrm{CO}}\right)$ for the whole velocity range and for the region covering the whole X-ray remnant was found to be $\sim 110 \mathrm{~K} \mathrm{~km} \mathrm{~s}^{-1}$. Since the CO sky maps are binned as $0.125 \times 0.125$, the area corresponding to the total $W_{\text {CO }}$ emission is $(0.125)^{2}$. We calculated $W_{\text {CO }}$ for each abovementioned velocity range and found that the highest contribution at the SNR's X-ray location came from the velocity range of $90-120 \mathrm{~km} \mathrm{~s}^{-1}$, which is also apparent in the CO sky maps in the blue framed panel in the bottom right corner of Figure 4.

When we calculated the integrated $\mathrm{CO}$ intensity over the velocity and extent of the cloud, $S_{\mathrm{CO}}$, we took all the velocity ranges into account: $S_{\mathrm{CO}}=\sum_{i}\left(W_{\mathrm{CO}} A\right)_{i}$, where $i$ represents the different velocity ranges. So, we found $S_{\mathrm{CO}}=1.72 \mathrm{~K} \mathrm{~km} \mathrm{~s}^{-1}$ $\mathrm{deg}^{2}$ for CO data used from the whole velocity range. For the dominant velocity range of $90-120 \mathrm{~km} \mathrm{~s}^{-1}$, we found $S_{\mathrm{CO}}=$ $1.11 \mathrm{~K} \mathrm{~km} \mathrm{~s}^{-1} \mathrm{deg}^{2}$. Assuming a linear relationship between the velocity-integrated $\mathrm{CO}$ intensity, $W_{\mathrm{CO}}$, and the molecular hydrogen column density, $N\left(\mathrm{H}_{2}\right)$ :

$$
\frac{N\left(\mathrm{H}_{2}\right)}{W_{\mathrm{CO}}}=(1.8 \pm 0.3) \times 10^{20} \mathrm{~cm}^{-2} \mathrm{~K}^{-1} \mathrm{~km} \mathrm{~s}^{-1}
$$

as given in Dame et al. (2001). Equation (1) gives

$$
\frac{M_{\mathrm{CO}}}{M_{\odot}}=1200 S_{\mathrm{CO}} d_{\mathrm{kpc}},
$$

where $d_{\mathrm{kpc}}$ is the distance to the cloud in kiloparsecs. We calculated the total hydrogen column density as $N\left(\mathrm{H}_{2}\right)=1.98 \times$ $10^{22} \mathrm{~cm}^{-2}$ using the $\mathrm{CO}$ data in the whole velocity range. For the velocity range of $90-120 \mathrm{~km} \mathrm{~s}^{-1}$ we obtained $N\left(\mathrm{H}_{2}\right)=1.28 \times$ $10^{22} \mathrm{~cm}^{-2}$. From Equation (2), the total mass of the clouds with velocities in the range of $90-120 \mathrm{~km} \mathrm{~s}^{-1}$ was found as $\mathrm{M}_{\mathrm{CO}}=$ $6.9 \times 10^{4} M_{\odot}$ using the lower limit on the distance to the cloud $(\sim 7.2 \mathrm{kpc})$. We estimated the size of the emission region as 15.7 pc. By assuming a spherical geometry of the cloud, we computed the density of the source region to be $4.25 M_{\odot} \mathrm{pc}^{-3}$ and the average density of protons to be 269 protons $\mathrm{cm}^{-3}$. Using the upper limit of the distance to the cloud $(\sim 11.4 \mathrm{kpc})$, we obtained an upper limit for the average proton density, 671 protons $\mathrm{cm}^{-3}$. Considering only the highest cloud velocity range, we recalculated the proton density as 173 and 435 protons $\mathrm{cm}^{-3}$ for the source distances of 7.2 and $11.4 \mathrm{kpc}$, respectively. Averaging the proton density over all different combinations of the distance and velocity parameters, we obtained 387 protons $\mathrm{cm}^{-3}$. Wilner et al. (1998) found $\sim 300$ protons $\mathrm{cm}^{-3}$, a value typical for giant MCs.

\subsubsection{Modeling and Interpretation}

To determine if the observed gamma-ray SED of 3C 391 can be fitted by a hadronic model, we fit the gamma-ray spectrum resulting from the decay of neutral pions, $\pi^{\circ}$, following Kelner et al. (2006). In order to calculate the gamma-ray spectrum, we considered that the relativistic protons follow a BPL-type spectrum: 


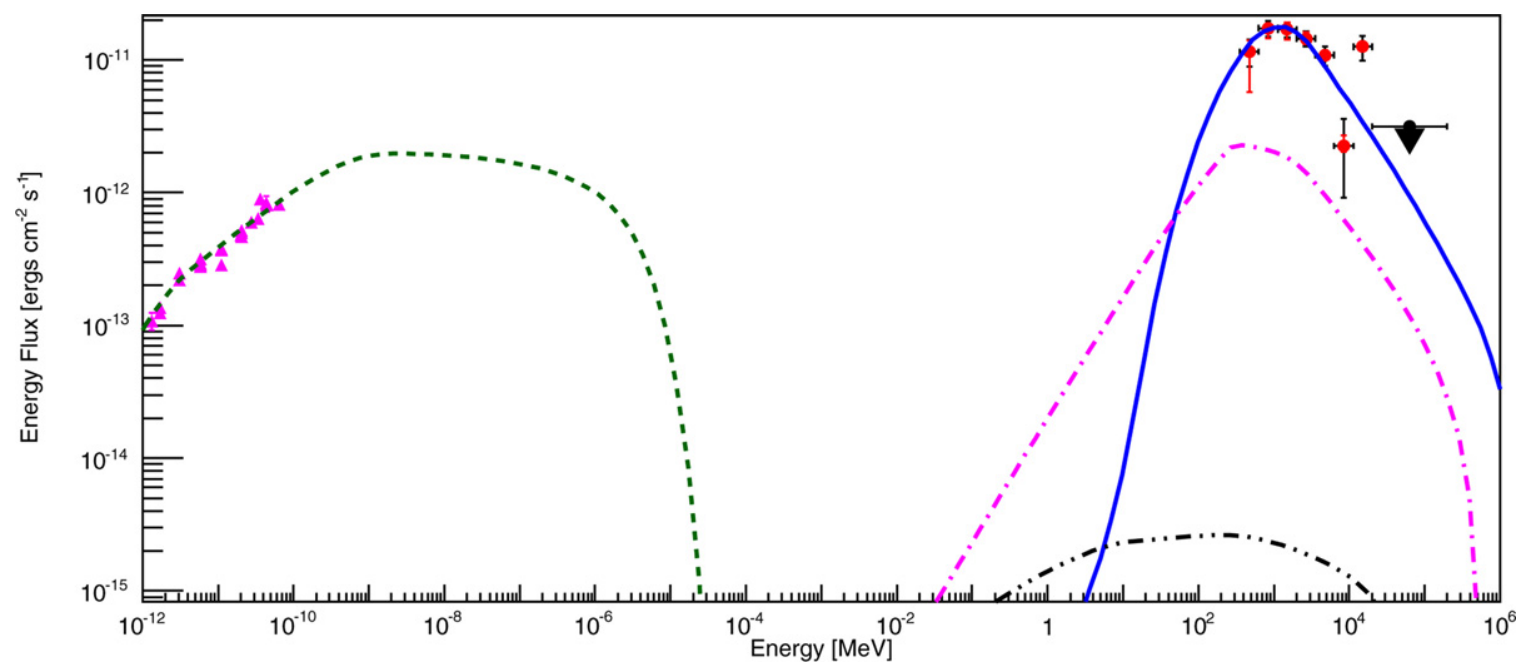

Figure 5. Models fit to the radio (Kassim 1989) (magenta filled triangles) and gamma-ray data (red filled circles with their corresponding statistical and systematic errors) are shown with solid blue ( $\pi^{0}$-decay spectral model component), green dashed (synchrotron emission), magenta dotted-dashed (non-thermal bremsstrahlung component), and black double-dot-dashed (IC emission component) lines.

(A color version of this figure is available in the online journal.)

$$
\begin{aligned}
\frac{d N}{d E_{p}} & =N_{1} E_{p}^{-\alpha} \text { for } E_{p}<E_{\mathrm{br}} \\
& =N_{2} E_{p}^{-\beta} \exp \left(-\frac{E_{p}}{E_{p_{\max }}}\right) \text { for } E_{\mathrm{br}} \leqslant E_{p} \leqslant E_{p_{\max }}
\end{aligned}
$$

In Equation (3), $E_{p}$ is the proton energy and $E_{\mathrm{br}}$ is the spectral break energy, where the spectral index changes from $\alpha$ to $\beta$. $E_{p_{\max }}$ is the maximum energy of protons and, during the fitting procedure, we assumed it to be at $10 \mathrm{TeV}$. $N_{1}$ and $N_{2}$ are normalization constants.

The best-fit parameters for the proton spectrum were obtained by a $\chi^{2}$-fitting procedure to the flux points. The estimated parameters are $\alpha=2.48 \pm 0.45, \beta=3.0 \pm 0.22$, and $E_{\mathrm{br}}=$ $12 \mathrm{GeV}$. The $\chi^{2} /$ dof is estimated to be $\simeq 1.6$. The best-fit gamma-ray spectrum resulting from the decay of neutral pions for an ambient gas density of $\sim 387 \mathrm{~cm}^{-3}$ is shown in Figure 2 with the blue solid line. The estimated total energy can be written as $W_{p} \simeq 5.81 \times 10^{48}\left(387 \mathrm{~cm}^{-3} / n_{H}\right) \mathrm{erg}$, where $n_{H}$ is the effective gas number density for $\mathrm{p}-\mathrm{p}$ collision. In addition to the BPL spectrum, different proton spectra, like PL, LP, and PLEC, were considered to explain the gamma-ray spectrum. However, we did not find any significant difference in the estimated best-fit parameters for all the input proton spectra.

To check the hadronic scenario from the energy point of view, we considered the energy from the $\mathrm{SN}$ explosion converted into accelerated protons, $W_{p}=\mathrm{L} \times \tau_{p}$, where $\mathrm{L}$ is the gammaray luminosity and $\tau_{p}$ is the characteristic cooling time of protons. When the gamma-ray luminosity is dominated by hadronic emission, then $\tau_{p}=5.3 \times 10^{7}\left(\mathrm{n} /\left(1 \text { proton } \mathrm{cm}^{-3}\right)\right)^{-1} \mathrm{yr}$ (Aharonian 2004). Using the average proton density of 387 protons $\mathrm{cm}^{-3}$ for $n$, we found $\tau_{p}=1.37 \times 10^{5} \mathrm{yr}$. So, taking $W_{p} \sim 5.81 \times 10^{48} \mathrm{erg}$, the luminosity of 3C 391 was found as $\mathrm{L}=1.34 \times 10^{36} \mathrm{erg} \mathrm{s}^{-1}$.

We also calculated the contribution from the leptonic emission models (Blumenthal \& Gould 1970). We found that the relativistic electrons cannot account for the gamma-ray spectrum at $\mathrm{GeV}$ energies through inverse Compton (IC) and bremsstrahlung processes. We assumed a BPL-type spectrum for electrons, which is similar to that considered for protons (Equation (3)).
The fit to the radio data (Kassim 1989) gave a synchrotron spectral index $\sigma \simeq 0.55\left(S_{v} \propto v^{-\sigma}\right)$. Therefore, we took $\alpha=2.1$ in the electron spectrum before the break because this parameter determines the shape of the synchrotron spectrum at radio wavelengths. On the other hand, $\beta$ can be found out from the fit to the observed gamma-ray spectrum. We considered an electron-toproton ratio of 0.01 following the observed spectra of the Galactic cosmic electrons and protons. We then considered the magnetic field and the number of electrons in the emission volume such that the synchrotron spectrum of electrons could explain the observed radio data as shown in Figure 5. To estimate the spectrum from leptonic models we used the following parameters: $\alpha=2.1, \beta=3.0, E_{\mathrm{br}}=7 \mathrm{GeV}, B=210 \mu \mathrm{G}, n=387 \mathrm{~cm}^{-3}$. We found the total energy of electrons as $W_{e}=1.4 \times 10^{47} \mathrm{erg}$. Assuming that the gamma rays at $\mathrm{GeV}$ energies are produced by the same population of electrons, we estimated the IC spectrum by taking the cosmic microwave background radiation and interstellar background radiation fields following Porter et al. (2008). We found that neither the IC nor the bremsstrahlung emission could account for the observed gamma-ray fluxes shown in Figure 5.

\subsection{X-Rays}

\subsubsection{Observation and Data Reduction}

3C 391 was observed with XIS on board Suzaku on 2010 October 22, under the observation ID of 505007010 and an exposure time of $\sim 99.4 \mathrm{ks}$. Detailed descriptions of the Suzaku satellite, the XIS instrument, and the X-ray telescope are given in Mitsuda et al. (2007), Koyama et al. (2007), and Serlemitsos et al. (2007), respectively. The XIS system consists of four CCD cameras (XIS 0,1,2, and 3). One of the cameras (XIS1) uses a back-illuminated (BI) CCD while the others (XIS0, 2, and 3) use front-illuminated (FI) CCDs. XIS2 has not been functional due to an unexpected anomaly in 2006 November. The XIS was operated in the normal full-frame clocking mode.

For the data reduction, we used HEASoft package version 6.11.1. The calibration database (CALDB: 20130305) was used and fitting was carried out in the X-ray spectral fitting package (xspec) version 11.3.2 (Arnaud 1996). The redistribution matrix files of the XIS were produced by xisrmfgen and auxiliary 


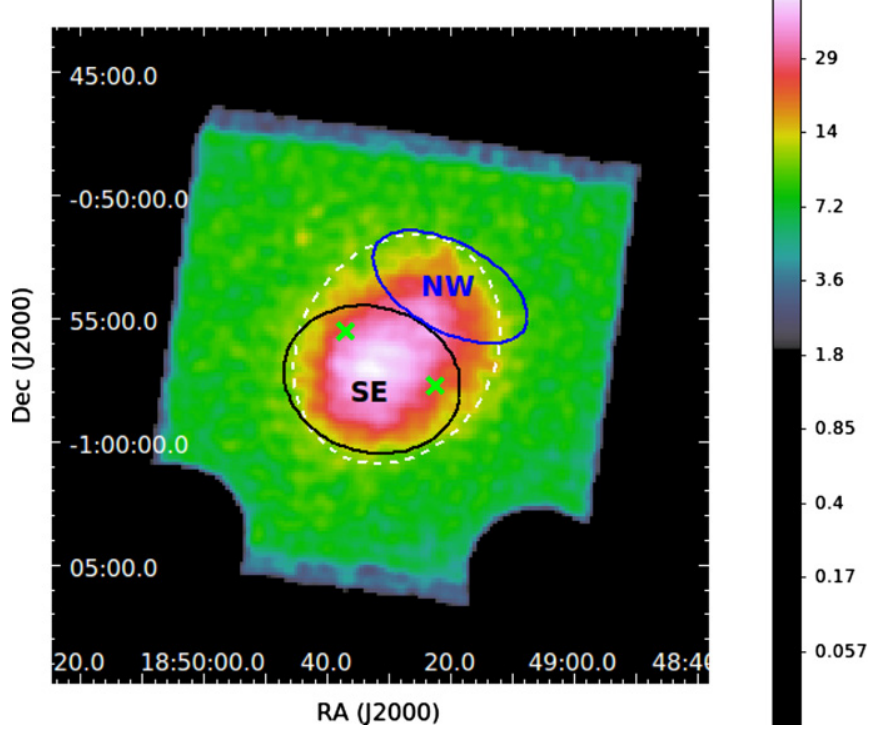

Figure 6. Suzaku XIS1 image of 3C 391 in the $0.3-10.0 \mathrm{keV}$ energy band. The whole SNR region shown with the white dashed ellipse and the NW and SE regions of the SNR shown with blue and black solid ellipses, respectively, are chosen for the spectral analysis. The two maser spots are represented with green crosses (Frail et al. 1996). The bottom corners irradiated by the calibration sources of ${ }^{55} \mathrm{Fe}$ are excluded.

(A color version of this figure is available in the online journal.)

response files were generated by xissimarfgen (Ishisaki et al. 2007).

\subsubsection{Background Estimation and Model Fitting}

For the spectral analysis of 3C 391, we selected three regions: the whole SNR and the NW and SE regions of the SNR. These regions are shown on the XIS1 image of $3 \mathrm{C}$ 391 in the $0.3-10.0 \mathrm{keV}$ energy band in Figure 6 as white dashed, blue solid, and black solid ellipses, respectively. The region representing the whole remnant has a size of $4.85 \times$ 3.94 centered at R.A. $(\mathrm{J} 2000)=18^{\mathrm{h}} 49^{\mathrm{m}} 28.6$, decl. $(\mathrm{J} 2000)=$ $-0^{\circ} 56^{\prime} 16^{\prime \prime} 4$. The reasons for this selection are given in the Discussion section.

The background for 3C 391 is a combination of the non-X-ray background (NXB), the cosmic X-ray background (CXB) emission, and the Galactic ridge X-ray emission, GRXE (Koyama et al. 1986). First, we estimated the NXB data from night Earth observations using the tool xisnxbgen (Tawa et al. 2008), and subtracted the NXB from the spectrum. We selected the background region, the nearby blank sky region (Obs.ID 500009020) on the Galactic plane, consisting of the GRXE and the CXB. The NXB-subtracted background spectrum was subtracted from the source spectrum using xspec. The spectrum was binned to a minimum of 20 counts bin $^{-1}$ using grppha to allow use of the $\chi^{2}$ statistic.

We first started the XIS analysis with the whole SNR region. We applied an absorbed (wabs in xspec; Morrison \& McCammon 1983) VNEI model for an NEI collisional plasma with variable abundances (Borkowski et al. 2001), which gave the reduced $\chi^{2}$ value of $950.2 / 659=1.44$ for the energy range of 1.0-5.0 keV. During model fitting, $N_{\mathrm{H}}, k T_{e}, n_{e} t$, and the abundances of $\mathrm{Mg}, \mathrm{Si}$, and $\mathrm{S}$ were free parameters, while the other elemental abundances were fixed to their solar values (Anders \& Grevesse 1989). Residuals of the VNEI spectral fit show that there is a clear residual emission at

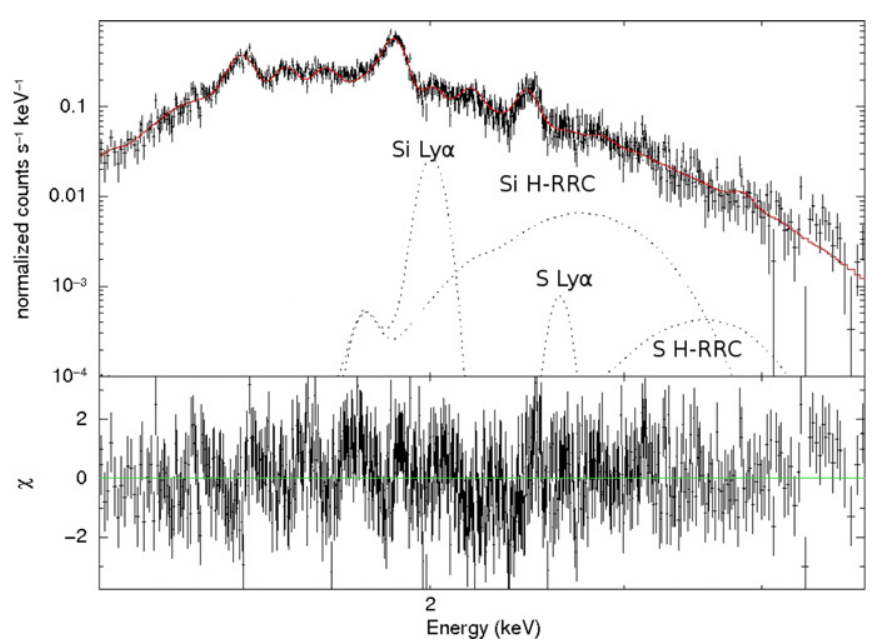

Figure 7. Background-subtracted FI spectrum of 3C 391 in the $1.0-5.0 \mathrm{keV}$ energy band fitted with an absorbed VNEI model with RRC and Ly $\alpha$ lines of the $\mathrm{Si}$ and $\mathrm{S}$ for the whole SNR. At the bottom of this panel, the residuals from the best-fit model are shown.

(A color version of this figure is available in the online journal.)

energies of $\sim 2.0 \mathrm{keV}$ and $\sim 2.6 \mathrm{keV}$. Therefore, we added two Gaussian components (the Gauss model in xspec) to the VNEI model. These two lines in the spectrum correspond to the H-like $(\mathrm{Ly} \alpha)$ lines of $\mathrm{Si}$ and $\mathrm{S}$, which are the indicators of highly ionized plasma. We note that we found Al K-shell emission at $\sim 1.58 \mathrm{keV}$ from this remnant, as it was also found for G344.7-0.1 (Yamaguchi et al. 2012), G350.1-0.3, and $\mathrm{G} 349.7+0.2$ (Yasumi et al. 2014). We follow the prescription described in Ozawa et al. (2009) for W49B to understand if the X-ray continuum comes from the thermal bremsstrahlung process or from RRC. We added the RRC model of H-like Si $(2.666 \mathrm{keV})$ and $\mathrm{S}(3.482 \mathrm{keV})$. The added Ly $\alpha$ lines and the RRC components improved the quality of the fit $\left(\chi^{2} /\right.$ dof $=$ $860 / 717)$. This suggests that these residuals are caused by the RRC of Si and S. Figure 7 shows the background-subtracted FI spectrum fitted with the absorbed VNEI plus RRC models and the Ly $\alpha$ lines of the Si and S, in the energy range of 1.0-5.0 keV. The same analysis steps described above were also applied for the NW and SE regions of 3C 391. The parameters $(90 \%$ confidence level) computed for the best-fit model obtained for the whole SNR and for the NW and SE regions are presented in Table 3.

\section{DISCUSSION}

In this paper, we showed that the gamma-ray spectrum of 3C 391 can be described by a hadronic emission model, which is clear evidence for acceleration of protons in this SNR. The neutral pion decay model assumed that the protons follow a BPL distribution with the $\alpha$ index standing for the acceleration of cosmic rays in the shock and the $\beta$ index representing the energy, above which protons escape from the SNR shell. Since the bremsstrahlung spectrum depends linearly on the number density of ambient matter, for 3C 391 it can only account for the observed spectrum at $\mathrm{GeV}$ energies, if $n=3000 \mathrm{~cm}^{-3}$. However, the spectrum has to be much steeper at $\sim 1 \mathrm{GeV}$ to explain the observed spectrum at this energy. Introducing an abrupt break at $\sim 800 \mathrm{MeV}$ in the electron spectrum can explain the observed fluxes at $\sim 1 \mathrm{GeV}$, but this break in the electron spectrum will make the synchrotron radio spectrum inefficient 
Table 3

Best-fit Spectral Parameters of 3C 391 with Corresponding Errors at the 90\% Confidence Level in the 1.0-5.0 keV Band for an Absorbed VNEI and RRC Models for Three Regions Shown in Figure 6

\begin{tabular}{|c|c|c|c|c|}
\hline Component & Parameters & Whole & NW & SE \\
\hline wabs & $N_{\mathrm{H}}\left(10^{22} \mathrm{~cm}^{-2}\right)$ & $3.1 \pm 0.1$ & $3.4 \pm 0.1$ & $2.9 \pm 0.1$ \\
\hline \multirow[t]{6}{*}{ VNEI } & $k T_{e}(\mathrm{keV})$ & $0.58 \pm 0.01$ & $0.61 \pm 0.01$ & $0.54 \pm 0.01$ \\
\hline & Mg (solar) & $1.2 \pm 0.1$ & $1.6 \pm 0.2$ & $1.4 \pm 0.1$ \\
\hline & Si (solar) & $0.9 \pm 0.1$ & $0.9 \pm 0.1$ & $1.1 \pm 0.1$ \\
\hline & $\mathrm{S}$ (solar) & $0.8 \pm 0.1$ & $0.7 \pm 0.1$ & $0.8 \pm 0.1$ \\
\hline & $\tau\left(10^{12} \mathrm{~cm}^{-3} \mathrm{~s}\right)$ & $1.8 \pm 0.2$ & $1.7 \pm 0.3$ & $1.8 \pm 0.1$ \\
\hline & Norm (photon $\mathrm{cm}^{-2} \mathrm{~s}^{-1}$ ) & $4.1 \pm 0.4$ & $3.1 \pm 0.5$ & $3.2 \pm 0.9$ \\
\hline \multirow[t]{3}{*}{$\mathrm{A} 1 \mathrm{~K} \alpha$} & $E(\mathrm{keV})$ & 1.58 (fixed) & 1.58 (fixed) & 1.58 (fixed) \\
\hline & $\sigma(\mathrm{keV})$ & 0 (fixed) & 0 (fixed) & 0 (fixed) \\
\hline & Norm $\left(10^{-4}\right.$ photon $\left.\mathrm{cm}^{-2} \mathrm{~s}^{-1}\right)$ & $2.1 \pm 0.2$ & $2.2 \pm 0.4$ & $2.1 \pm 0.1$ \\
\hline \multirow[t]{3}{*}{ Si Ly $\alpha$} & $E(\mathrm{keV})$ & 2.0 (fixed) & 2.0 (fixed) & 2.0 (fixed) \\
\hline & $\sigma(\mathrm{keV})$ & 0 (fixed) & 0 (fixed) & 0 (fixed) \\
\hline & Norm $\left(10^{-4}\right.$ photon $\left.\mathrm{cm}^{-2} \mathrm{~s}^{-1}\right)$ & $4.9 \pm 0.5$ & $3.8 \pm 0.3$ & $3.9 \pm 0.2$ \\
\hline \multirow[t]{3}{*}{$\operatorname{S~Ly~} \alpha$} & $E(\mathrm{keV})$ & 2.6 (fixed) & 2.6 (fixed) & 2.6 (fixed) \\
\hline & $\sigma(\mathrm{keV})$ & 0 (fixed) & 0 (fixed) & 0 (fixed) \\
\hline & Norm $\left(10^{-4}\right.$ photon $\left.\mathrm{cm}^{-2} \mathrm{~s}^{-1}\right)$ & $3.6 \pm 0.2$ & $3.1 \pm 0.3$ & $2.9 \pm 0.2$ \\
\hline \multirow[t]{2}{*}{ RRC H-like Si } & $E(\mathrm{keV})$ & 2.666 (fixed) & 2.666 (fixed) & 2.666 (fixed) \\
\hline & Norm $\left(10^{-4}\right.$ photon $\left.\mathrm{cm}^{-2} \mathrm{~s}^{-1}\right)$ & $5.2 \pm 0.3$ & $4.3 \pm 0.6$ & $4.1 \pm 0.3$ \\
\hline \multirow[t]{3}{*}{ RRC H-like S } & $E(\mathrm{keV})$ & 3.482 (fixed) & 3.482 (fixed) & 3.482 (fixed) \\
\hline & Norm $\left(10^{-4}\right.$ photon $\left.\mathrm{cm}^{-2} \mathrm{~s}^{-1}\right)$ & $4.4 \pm 0.5$ & $3.7 \pm 0.4$ & $3.6 \pm 0.2$ \\
\hline & $\chi^{2} /$ dof & $860 / 717=1.2$ & $452 / 361.6=1.25$ & $301 / 251=1.2$ \\
\hline
\end{tabular}

for explaining the observed radio fluxes. In the case of the IC emission process, the total energy has to be $\sim 10^{51}$ erg to account for the observed spectrum. This means that almost all the energy released during the $\mathrm{SN}$ explosion has been transferred to the relativistic electrons, which is very unlikely. Moreover, the magnetic field needs to be $\leqslant 1 \mu \mathrm{G}$ to explain the radio data. Additionally, the density of the ambient matter has to be $\sim 0.3 \mathrm{~cm}^{-3}$ to reduce the bremsstrahlung component, which will be inconsistent with our measured value of $387 \mathrm{~cm}^{-3}$.

The total gamma-ray luminosity was found to be $L=1.34 \times$ $10^{36} \mathrm{erg} \mathrm{s}^{-1}$, similar to the first GeV-emitting SNRs that were discovered by Fermi-LAT, e.g., IC443 (Abdo et al. 2010b), W51C (Abdo et al. 2009b), W44 (Abdo et al. 2010d), and W49B (Abdo et al. 2010e), all of which are MCs interacting with MM SNRs with gamma-ray luminosities higher than $10^{35} \mathrm{erg} \mathrm{s}^{-1}$.

There are mainly two scenarios describing how hadronic gamma rays are produced in SNRs. The "crushed cloud" scenario describes the hadronic gamma rays as a product of interactions between the MC, compressed and shocked by the passage of the blast-wave of the SNR, and the relativistic protons inside the shocked MC. The relativistic protons can be either reaccelerated cosmic rays or freshly accelerated protons entering the radiatively compressed MC. In this scenario, since the crushed clouds are thin, multi-GeV particles can escape from the shocked MCs, which might be the reason for seeing a break in the proton spectrum (Blandford \& Cowie 1982; Uchiyama et al. 2010). In the "escaped cosmic rays" scenario, escaping relativistic protons reach a nearby unshocked $\mathrm{MC}$ and produce $\pi^{\circ}$-decay gamma rays. For this scenario to happen, there must be $\mathrm{GeV} / \mathrm{TeV}$ sources found outside the radio shell of 3C 391 that could produce these escaping protons (Gabici et al. 2009). However, there are no nearby cosmic ray sources for 3C 391 and all other sources were taken into account in the gamma-ray background model.

The reason why the spectral break of protons is at $\sim 12 \mathrm{GeV}$ for 3C 391 could be that relativistic particles are escaping from their acceleration sites, the shell of the SNR, or the crushed MC when the shell is expanding into the rarefied ISM during the earlier epochs of the SNR. Since particles at very high energies $(\sim \mathrm{TeV})$ can only be confined during the early stages of the SNR evolution, and because 3C 391 is a middle-aged SNR, most of the very high energy particles must have already escaped from the shell (Uchiyama et al. 2010). The interactions between the SNR and MC, which are evident from $\mathrm{OH}$ masers (Frail et al. 1996), can enhance particle escape. Alternatively, the SNR shock expanding in dense medium can be slowed down by the dense MC shifting the maximum particle energy to the $\mathrm{GeV}$ region (Ohira et al. 2011). Assuming a BPL spectrum of protons without any spectral cutoff, we estimate that the differential flux of gamma rays at $1 \mathrm{TeV}$ is $\sim 0.06 \%$ of the Crab nebula flux. $\mathrm{TeV}$ observations of this source with the upcoming Cherenkov Telescope Array may provide more robust constraints on the various parameters of the input proton spectrum.

The massive progenitor star of 3C 391 inside an MC exploded and the shock waves expanded in the dense MC, breaking out into a more rarefied ISM in the SW region, where most of the very high energy particles escaped, causing a break in the proton spectrum. This "break-out" scenario could also be an explanation for observing RP in 3C 391. Using Suzaku data, we discovered RRC of $\mathrm{H}$-like $\mathrm{Si}$ and $\mathrm{S}$ at $\sim 2.7$ and $\sim 3.5 \mathrm{keV}$ from the spectrum.

We chose three regions in which to do X-ray spectral analysis to compare the $k T_{e}$ values in different regions of 3C 391: the whole SNR, the NW region, and the SE region (Figure 6). We chose the NW and SE regions to check if there is any temperature gradient across the SNR. Finding a temperature gradient would be a sign for electron cooling through a thermal conduction mechanism. The NW region is closer to the site of denser molecular material, where the molecular density drops gradually toward the SE of 3C 391 (Wilner et al. 1998). This is a good region in which to test the thermal conduction scenario of the RP. The SE region includes two $\mathrm{OH}$ maser spots, an 
indication that the SNR shell is breaking out of the MC and into the rarefied ISM (Frail et al. 1996). By choosing this region, we aim to check the adiabatic cooling scenario over ionized plasma. When we compared the $k T_{e}$ values of these regions, we found $k T_{e}$ for $\mathrm{NW}$ and $\mathrm{SE}$ region to be $\sim 0.61 \mathrm{keV}$ and $\sim 0.54 \mathrm{keV}$, respectively. Since these values are very close to each other, it is not possible to determine which cooling mechanism is dominant.

Analyzing X-rays from the whole remnant and assuming a distance of $7.2 \mathrm{kpc}$ for 3C 391 (Radhakrishnan et al. 1972), we calculated the electron density $n_{\mathrm{e}}$ as $\sim 0.82 \mathrm{~cm}^{-3}$ from the emission measure $\left(\mathrm{EM}=n_{\mathrm{e}} n_{\mathrm{H}} V\right.$, where $n_{\mathrm{H}}$ is the hydrogen density and $V$ is the volume of the X-ray-emitting plasma). Then, from the relation $\tau / n_{\mathrm{e}}$, we found the age of 3C 391 as $\sim 69,000$ yr using the best-fit $\tau$ value $\left(\sim 1.8 \times 10^{12} \mathrm{~cm}^{-3} \mathrm{~s}\right)$ of the VNEI plus RRC model, which is higher than the SNR age found by Chandra (Chen et al. 2004).

The possible origin of the RP found in the NW region might be due to hot electrons coming into contact with cooler and denser $\mathrm{MCs}$, impeding the expansion of the SNR shell. In the SE region of 3C 391, it is possible that the RP formed when a SN blast wave expanded into the rarefied ISM and caused the electron temperature to drop through the adiabatic cooling mechanism. Both of these cooling mechanisms might have worked together in different regions of the SNR to produce RRC in 3C 391. To understand which scenario dominates in which part of 3C 391, detailed over-ionization maps need to be produced, as was done for W49B (Lopez et al. 2013).

\section{CONCLUSION}

We have analyzed $\mathrm{GeV}$ gamma rays from 3C 391 and the spectrum revealed that the emission is most likely hadronic in origin. The gamma-ray spectrum follows the spectrum of parent protons, a BPL distribution with spectral index parameters of $\alpha=2.48$ and $\beta=3.0$ and a spectral break at $\sim 12 \mathrm{GeV}$. This suggests that protons are accelerated to high energies, possibly at the region of a SNR shell breaking through the shocked MC into the rarefied ISM. The low breaking energy can be explained either by higher energy protons having already escaped through the thin crushed MC or the SNR shell at the earlier evolutionary stages of the SNR, or by the SNR shock expanding in a dense medium being slowed down by this medium. The first model can be applicable especially to the SE region of 3C 391 where a break-out morphology is evident from the radio data, while the latter one is more suitable to the NW region of 3C 391 where the cloud material becomes denser and cooler.

We also studied the plasma structure of the MM SNR 3C 391 using Suzaku XIS data. The X-ray spectra of the SNR are well represented with a VNEI plus RRC model. We discovered RRC of H-like Si and S at $\sim 2.7$ and $\sim 3.5 \mathrm{keV}$. We discussed the possible electron cooling mechanisms for 3C 391 and we concluded that both thermal conduction and adiabatic cooling scenarios are possible explanations for the existence of RRC found in 3C 391.

We thank the referee for the detailed and constructive comments and suggestions on the manuscript. We are also grateful to the Fermi-LAT collaboration members for their support. Special thanks go to J. Lande for his helpful comments on pointlike and to L. Tibaldo for his insightful suggestions about the Fermi-LAT analysis. T. Ergin acknowledges support from the Scientific and Technological Research Council of Turkey (TÜBITAK) through the BIDEB-2232 fellowship program.
E. N. Ercan thanks Bogazici University for the financial support through the BAP project-5052.

Facilities: Fermi, Suzaku

\section{REFERENCES}

Abdo, A. A., Ackermann, M., Ajello, M., et al. 2009a, ApJS, 183, 46 Abdo, A. A., Ackermann, M., Ajello, M., et al. 2009b, ApJL, 706, L1 Abdo, A. A., Ackermann, M., Ajello, M., et al. 2010a, ApJS, 187, 460 Abdo, A. A., Ackermann, M., Ajello, M., et al. 2010b, ApJ, 712, 459 Abdo, A. A., Ackermann, M., Ajello, M., et al. 2010c, ApJ, 718, 348 Abdo, A. A., Ackermann, M., Ajello, M., et al. 2010d, Sci, 327, 1103 Abdo, A. A., Ackermann, M., Ajello, M., et al. 2010e, ApJ, 722, 1303 Ackermann, M., Ajello, M., Allafort, A., et al. 2013, Sci, 339, 807

Aharonian, F. A. 2004, Very High Energy Cosmic Gamma Radiation: A Crucial Window on the Extreme Universe (River Edge, NJ: World Scientific) Anders, E., \& Grevesse, N. 1989, GeCoA, 53, 197

Arnaud, K. A. 1996, in ASP Conf. Ser. 101, Astronomical Data Analysis Software and Systems V, ed. G. H. Jacoby \& J. Barnes (San Francisco, CA: ASP), 17

Atwood, W. B., Abdo, A. A., Ackermann, M., et al. 2009, ApJ, 697, 1071

Blandford, R. D., \& Cowie, L. L. 1982, ApJ, 260, 625

Blumenthal, G. R., \& Gould, R. J. 1970, RvMP, 42, 237

Bochow, A. 2011, in Proc. of ICRC 2011, Vol. 7, 110

Borkowski, K. J., Lyerly, W. J., \& Reynolds, S. P. 2001, ApJ, 548, 820

Castro, D., \& Slane, P. 2010, ApJ, 717, 372

Castro, D., Slane, P. O., Carlton, A., \& Figueroa-Feliciano, E. 2013, ApJ, 774,36

Chen, Y., \& Slane, P. O. 2001, ApJ, 563, 202

Chen, Y., Su, Y., Slane, P. O., \& Wang, Q. D. 2004, ApJ, 616, 885

Claussen, M. J., Frail, D. A., Goss, W. M., \& Gaume, R. A. 1997, ApJ, 489, 143

Cox, D. P., Shelton, R. L., Maciejewski, W., et al. 1999, ApJ, 524, 179

Dame, T. M., Hartmann, D., \& Thaddeus, P. 2001, ApJ, 547, 792

Dame, T. M., Ungerechts, H., Cohen, R. S., et al. 1987, ApJ, 322, 706

Frail, D. A., Goss, W. M., Reynoso, E. M., et al. 1996, AJ, 111, 1651

Gabici, S., Aharonian, F. A., \& Casanova, S. 2009, MNRAS, 396, 1629

Green, A. J., Frail, D. A., Goss, W. M., \& Otrupcek, R. 1997, AJ, 114, 2058

Hewitt, J. W., \& Yusef-Zadeh, F. 2009, ApJL, 694, L16

Hewitt, J. W., Yusef-Zadeh, F., \& Wardle, M. 2008, ApJ, 683, 189

Ishisaki, Y., Maeda, Y., Fujimoto, R., et al. 2007, PASJ, 59, 113

Itoh, H., \& Masai, K. 1989, MNRAS, 236, 885

Kassim, N. E. 1989, ApJS, 71, 799

Kawasaki, M., Ozaki, M., Nagase, F., Inoue, H., \& Petre, R. 2005, ApJ, 631,935

Kawasaki, M., Ozaki, M., Nagase, F., et al. 2002, ApJ, 572, 897

Kelner, S. R., Aharonian, F. A., \& Bugayov, V. V. 2006, PhRvD, 74, 034018

Keohane, J. W., Reach, W. T., Rho, J., \& Jarrett, T. H. 2007, ApJ, 654, 938

Kerr, M. 2011, arXiv:1101.6072

Koyama, K., Makishima, K., Tanaka, Y., \& Tsunemi, H. 1986, PASJ, 38, 121

Koyama, K., Tsunemi, H., Dotani, T., et al. 2007, PASJ, 59, 23

Lande, J., Ackermann, M., Allafort, A., et al. 2012, ApJ, 756, 5

Lopez, L. A., Pearson, S., Ramirez-Ruiz, E., et al. 2013, ApJ, 777, 145

Mitsuda, K., Bautz, M., Inoue, H., et al. 2007, PASJ, 59, 1

Moffett, D. A., \& Reynolds, S. P. 1994, ApJ, 425, 668

Moriya, T. J. 2012, ApJL, 750, L13

Morrison, R., \& McCammon, D. 1983, ApJ, 270, 119

Nolan, P. L., Abdo, A. A., Ackermann, M., et al. 2012, ApJS, 199, 31

Ohira, Y., Murase, K., \& Yamazaki, R. 2011, MNRAS, 410, 1577

Ohnishi, T., Koyama, K., Tsuru, T. G., et al. 2011, PASJ, 63, 527

Orlando, S., Bocchino, F., Reale, F., Peres, G., \& Pagano, P. 2008, ApJ, 678,274

Ozawa, M., Koyama, K., Yamaguchi, H., Masai, K., \& Tamagawa, T. 2009, ApJL, 706, L71

Porter, T. A., Moskalenko, I. V., Strong, A. W., Orlando, E., \& Bouchet, L. 2008, ApJ, 682, 400

Radhakrishnan, V., Goss, W. M., Murray, J. D., \& Brooks, J. W. 1972, ApJS, 24, 49

Reach, W. T., \& Rho, J. 1999, ApJ, 511, 836

Reach, W. T., Rho, J., Jarrett, T. H., \& Lagage, P.-O. 2002, ApJ, 564, 302

Reach, W. T., \& Rho, J.-H. 1996, A\&A, 315, L277 (Paper I)

Reynolds, S. P., \& Moffett, D. A. 1993, AJ, 105, 2226

Rho, J., \& Petre, R. 1998, ApJL, 503, L167

Rho, J.-H., \& Petre, R. 1996, ApJ, 467, 698

Sawada, M., \& Koyama, K. 2012, PASJ, 64, 81

Serlemitsos, P. J., Soong, Y., Chan, K.-W., et al. 2007, PASJ, 59, 9 
Shelton, R. L., Cox, D. P., Maciejewski, W., et al. 1999, ApJ, 524, 192

Shimizu, T., Masai, K., \& Koyama, K. 2012, PASJ, 64, 24

Su, Y., \& Chen, Y. 2005, ChJAA, 5, 412

Tawa, N., Hayashida, K., Nagai, M., et al. 2008, PASJ, 60, S11

Uchida, H., Koyama, K., Yamaguchi, H., et al. 2012, PASJ, 64, 141

Uchiyama, Y., Blandford, S., Funk, S., Tajima, H., \& Tanaka, T. 2010, ApJL, 723, L122
Wang, Z. R., \& Seward, F. D. 1984, ApJ, 279, 705

Wilner, D. J., Reynolds, S. P., \& Moffett, D. A. 1998, AJ, 115, 247

Yamaguchi, H., Ozawa, M., Koyama, K., et al. 2009, ApJL, 705, L6

Yamaguchi, H., Tanaka, M., Maeda, K., et al. 2012, ApJ, 749, 137

Yamauchi, S., Nobukawa, M., Koyama, K., \& Yonemori, M. 2013, PASJ, 65, 6

Yasumi, M., Nobukawa, M., Nakashima, S., et al. 2014, arXiv:1403.6898

Yusef-Zadeh, F., Uchida, K. I., \& Roberts, D. 1995, Sci, 270, 1801 